\title{
COMPLEX BOUNDARY VALUE PROBLEMS
}

\author{
BY \\ P. R. GARABEDIAN AND D. C. SPENCER
}

1. Introduction. In this paper we study the theory of functions of several complex variables from the point of view of partial differential equations of elliptic type. The basic objective is to obtain a generalization of the tools of potential theory which will apply to the Cauchy-Riemann equations in several variables. Thus we investigate further the kernel function and its relation to the Green's function introduced earlier $[8 ; 9]$.

Our point of departure here is a discussion of boundary value problems for harmonic differential forms in Euclidean space carried through by Hodge [12]. We find that in terms of two new complex differential operators $\bar{d}$ and $\bar{\delta}$ one can develop a theory of boundary value problems, analogous formally to Hodge's work, which centers about the Cauchy-Riemann equations. We are enabled to bring together systematically and concisely the concepts of the Cauchy formula, the Green's and Neumann's forms, the kernel form, and harmonic integrals, and to build from them an elegant generalization for several complex variables of the basic ideas of potential theory.

A number of new phenomena arise for the complex differential forms of our theory. While we rely strongly on the theory of the Fredholm integral equation, our boundary value problems lead to singular equations with infinitely many eigenfunctions. Thus to obtain a complete existence proof, we resort to a combination of the method of integral equations and a procedure of orthogonal projection developed around the Bergman kernel function [10]. Indeed, it is the recent results relating the kernel function to the Green's function which motivate our entire presentation. Thus we obtain a single theory which connects harmonic forms, integral equations, Cauchy's formula, and the kernel function in terms of suitable boundary value problems.

Many special cases and examples of our general theory prove to be of particular interest. A few of these are given special attention in a final section of the paper.

2. Definitions, notation, and formalism. We shall consider the $2 k$-dimensional Euclidean space of $k$ complex variables $z_{j}=x_{j}+i y_{j}$ and a cell $M$ in that space with smooth boundary $\partial M$. We shall set $y_{j}=x_{k+j}$, and

$$
\frac{\partial}{\partial z_{j}}=\frac{1}{2}\left(\frac{\partial}{\partial x_{j}}-i \frac{\partial}{\partial y_{j}}\right), \quad \frac{\partial}{\partial \bar{z}_{j}}=\frac{1}{2}\left(\frac{\partial}{\partial x_{i}}+i \frac{\partial}{\partial y_{j}}\right),
$$

Presented to the Society, September 7, 1951; received by the editors July 12, 1951. 
while $d z_{j}=d x_{j}+i d y_{j}, d \bar{z}_{j}=d x_{j}-i d y_{j}$.

In the formalism of E. Cartan, one introduces the differential form

$$
d x_{1} \cdots d x_{2 k}=\left(\frac{i}{2}\right)^{k} d z_{1} \cdots d z_{k} d \bar{z}_{1} \cdots d \bar{z}_{k}
$$

as volume element. The expression

$$
\phi_{p}=a_{i_{1}} \cdots i_{p} d z_{i_{1}} \cdots d z_{i_{p}}
$$

will be called a pure $p$-form, where summation over all combinations of indices with $i_{1}<i_{2}<\ldots<i_{p}$ is to be understood. In particular, with

$$
r^{2}=\sum_{j=1}^{k}\left|z_{j}-t_{j}\right|^{2}
$$

we introduce the singular $p$-form

$$
\omega_{p}(z, t)=\frac{1}{r^{2 k-2}} d z_{i_{1}} \cdots d z_{i_{p}} d \bar{t}_{i_{1}} \cdots d \bar{t}_{i_{n}}
$$

For pure forms $\phi_{p}$, the conjugation operator ${ }^{*}$ yields

$$
* \phi_{p}=\frac{2^{p}}{(2 i)^{k}} a_{i_{1}} \cdots i_{p} d \bar{z}_{i_{p+1}} \cdots d \bar{z}_{i_{k}} d z_{1} \cdots d z_{k},
$$

where $i_{1}, \cdots, i_{k}$ is an even permutation of the indices $1, \cdots, k$. In particu. lar (without summation with respect to $i_{1} i_{2} \cdots i_{p}$ ),

$$
d z_{i_{1}} \cdots d z_{i_{p} *}\left(d z_{i_{1}} \cdots d z_{i_{p}}\right)^{-}=2^{p} d x_{1} \cdots d x_{2 k},
$$

and

$$
* * \phi_{p}=(-1)^{p} \phi_{p}
$$

Also,

$$
{ }^{*} d \bar{z}_{i_{p+1}} \cdots d \bar{z}_{i_{k}} d z_{1} \cdots d z_{k}=(2 i)^{k}(-2)^{-p} d z_{i_{1}} \cdots d z_{i_{p}} .
$$

In addition to the usual differential operators $d$ and $\delta=* d^{*}$ of Hodge [13], we define here the complex operators $\bar{d}$ and $\bar{\delta}$ by the formulas

$$
\begin{aligned}
\bar{d} \phi_{p} & =\sum_{m=p+1}^{k} \frac{\partial a_{i_{1} \cdots i_{p}}}{\partial z_{i_{m}}} d z_{i_{m}} d z_{i_{1}} \cdots d z_{i_{p}}, \\
\bar{\delta} \phi_{p}=\frac{1}{2} * d * \phi_{p} & =\sum_{m=1}^{p}(-1)^{m+1} \frac{\partial a_{i_{1}} \cdots i_{p}}{\partial \bar{z}_{i_{m}}} d z_{i_{1}} \cdots\left[d z_{i_{m}}\right] \cdots d z_{i_{p}},
\end{aligned}
$$

where $\left[d z_{i_{m}}\right]$ indicates that this differential is to be omitted. Note that with $\zeta=d \bar{z}_{1} \cdots d \bar{z}_{k}$ we obtain 


$$
\zeta \bar{d} \phi_{p}=\zeta d \phi_{p}=(-1)^{k} d\left(\zeta \phi_{p}\right) .
$$

Also, $\bar{d} \bar{d} \phi_{p}=\bar{\delta} \bar{\delta} \phi_{p}=0$. The importance of the operators $\bar{d}$ and $\bar{\delta}$ stems from the fact that the Cauchy-Riemann equations for a function $f$ analytic in the $k$ complex variables $z_{1}, \cdots, z_{k}$ can be written $\bar{\delta}\left(f d z_{1} \cdots d z_{k}\right)=0$ or $\bar{d} \bar{f}=0$.

Two identities will be of fundamental importance in our work. They are

$$
\Delta \phi_{p}=\frac{1}{4} \sum_{m=1}^{2 k} \frac{\partial^{2} a_{i_{1}} \cdots i_{p}}{\partial x_{m}^{2}} d z_{i_{1}} \cdots d z_{i_{p}}=(\bar{d} \bar{\delta}+\bar{\delta} \bar{d}) \phi_{p}
$$

and

$$
\bar{d} \omega_{p}(z, t)=-\left(\bar{\delta} \omega_{p+1}(t, z)\right)^{-},
$$

where in the last relation $\bar{d}$ refers to differentiation with respect to $z$ variables and $\bar{\delta}$ refers to differentiation with respect to $t$ variables. For some positive constant $\sigma_{k}$ one obtains by Poisson's equation the further identity

$$
\phi_{p}(z)=-2^{-p} \sigma_{k} \Delta_{z} \int_{M} \phi_{p}(t) *(t)\left(\omega_{p}(t, z)\right)^{-} .
$$

We shall have need for the scalar product

$$
\left(\phi_{p}, \psi_{p}\right)=\int_{M} \phi_{p} *\left(\psi_{p}\right)^{-}
$$

of a pair of pure forms $\phi_{p}, \psi_{p}$. Green's theorem yields for this the relations

$$
\begin{aligned}
\left(\bar{d} \phi_{p}, \psi_{p+1}\right)+2\left(\phi_{p}, \bar{\delta} \psi_{p+1}\right) & =\int_{\partial M} \phi_{p} *\left(\psi_{p+1}\right)^{-} \\
2\left(\bar{\delta} \psi_{p+1}, \phi_{p}\right)+\left(\psi_{p+1}, \bar{d} \phi_{p}\right) & =\int_{\partial M}\left(* \psi_{p+1}\right)\left(\phi_{p}\right)^{-} .
\end{aligned}
$$

Similar identities can be obtained in curved cells $M$ by Stokes' theorem.

Finally, one obtains by a ready calculation

$$
\left[*\left(\zeta \phi_{p}\right)\right] \bar{\zeta}=(-2)^{k} * \phi_{p} .
$$

At a point of $\partial M$, one can introduce tangential coordinates $s_{1}, \cdots, s_{2 k-1}$ and a normal coordinate $n$. Then $\phi_{p}$ can be written

$$
\phi_{p}=\alpha_{i_{1}} \cdots i_{p} d s_{i_{1}} \cdots d s_{i_{p}}+\beta_{i_{1} \cdots i_{p-1}} d n d s_{i_{1}} \cdots d s_{i_{p-1}} .
$$

We define

$$
T \phi_{p}=\alpha_{i_{1}} \cdots i_{p} d s_{i_{1}} \cdots d s_{i_{p}} .
$$

3. Combined method of integral equations and orthogonal projection. Suppose that 


$$
\theta_{k+p}=T\left[\alpha_{i_{1}} \cdots i_{p} d z_{i_{1}} \cdots d z_{i_{p}} d \bar{z}_{1} \cdots d \bar{z}_{k}\right]
$$

is a given form on $\partial M$. We shall prove in this section that there exists in $M$ a unique pure form $A_{p}$ such that

$$
\begin{aligned}
T\left(\zeta A_{p}\right) & =\theta_{k+p}, \\
\bar{\delta} \bar{d} A_{p} & =0, \\
A_{p} & =\bar{\delta} Q_{p+1},
\end{aligned}
$$$$
\text { on } \partial M \text {, }
$$
in $M$, in $M$,

for some pure form $Q_{p+1}$. Our method of proof is based on the classical procedure of integral equations together with an application of a method of orthogonal projection developed recently in connection with the theory of kernel functions [10]. The result will serve as a basis for the study of analytic functions of several complex variables by the methods of the Fredholm integral equation, of harmonic forms, and of the general theory of partial differential equations of elliptic type.

Let $\phi_{p}$ be a given pure form defined in the neighborhood of $\partial M$, and let this form be extended into $M$ in some manner. Then by Poisson's equation

$$
-2^{-p} \sigma_{k} \Delta \int_{M} \phi_{p^{*}}\left(\omega_{p}\right)^{-}= \begin{cases}\phi_{p}, & \text { in } M, \\ 0, & \text { outside } M .\end{cases}
$$

Also,

$$
\begin{aligned}
-2^{-p} \sigma_{k} \Delta \int_{M} \phi_{p^{*}}\left(\omega_{p}\right)^{-} & =-2^{-p} \sigma_{k} \bar{\delta} \bar{d} \int_{M} \phi_{p} *\left(\omega_{p}\right)^{-}-2^{-p} \sigma_{k} \bar{d} \bar{\delta} \int_{M} \phi_{p} *\left(\omega_{p}\right)^{-} \\
& =2^{-p} \sigma_{k} \bar{\delta} \int_{M} \phi_{p} *\left(\bar{\delta} \omega_{p+1}\right)^{-}+2^{-p} \sigma_{k} \bar{d} \int_{M} \phi_{p} *\left(\bar{d} \omega_{p-1}\right)^{-} .
\end{aligned}
$$

Thus from Green's theorem

$$
\begin{aligned}
& 2^{-p} \sigma_{k} \bar{\delta}\left(\phi_{p}, \bar{\delta} \omega_{p+1}\right)+2^{-p} \sigma_{k} \bar{d}\left(\phi_{p}, \bar{d} \omega_{p-1}\right) \\
&=2^{-p-1} \sigma_{k} \bar{\delta} \int_{\partial M} \phi_{p}\left(\omega_{p+1}\right)^{-}+2^{-p} \sigma_{k} \bar{d} \int_{\partial M}\left(* \phi_{p}\right)\left(\omega_{p-1}\right)^{-} \\
& \\
& \quad-2^{-p-1} \sigma_{k} \bar{\delta}\left(\bar{d} \phi_{p}, \omega_{p+1}\right)-2^{-p+1} \sigma_{k} \bar{d}\left(\bar{\delta} \phi_{p}, \omega_{p-1}\right) \\
&= \begin{cases}\phi_{p}, & \text { in } M, \\
0, & \text { outside } M .\end{cases}
\end{aligned}
$$

Now $T d\left(\zeta \omega_{p-1}\right)=(-1)^{k} T \zeta \bar{d} \omega_{p-1}$, and it may be verified that

$$
T \zeta 2^{-p} \sigma_{k} \bar{d} \int_{\partial M}\left(* \phi_{p}\right)\left(\omega_{p-1}\right)^{-}
$$

is continuous across $\partial M$. Thus 


$$
T \zeta 2^{-p-1} \sigma_{k} \bar{\delta} \int_{\partial M} \phi_{p} *\left(\omega_{p+1}\right)^{-}
$$

must jump by $T \zeta \phi_{p}$ on $\partial M$.

If we set

$$
A_{p}=2^{-p-1} \sigma_{k} \bar{\delta} \int_{\partial M} \phi_{p} *\left(\omega_{p+1}\right)^{-},
$$

we find, by the usual argument, that $T \zeta A_{p}$ has on $\partial M$ the boundary values

$$
T \zeta A_{p}=P\left[T 2^{-p-1} \sigma_{k} \zeta \bar{\delta} \int_{\partial M} \phi_{p} *\left(\omega_{p+1}\right)^{-}\right]+\frac{1}{2} T \zeta \phi_{p},
$$

where $P[$ ] indicates that the integral inside the brackets is to be taken in the sense of the Cauchy principal value. Clearly

$$
\bar{\delta} \bar{d} A_{p}=2^{-p-1} \sigma_{k} \bar{\delta} \bar{d} \delta \int_{\partial M} \phi_{p} *\left(\omega_{p+1}\right)^{-}=2^{-p-1} \sigma_{k} \bar{\delta} \Delta \int_{\partial M} \phi_{p} *\left(\omega_{p+1}\right)^{-}=0,
$$

since $\Delta \omega_{p+1}=0$. Thus $A_{p}$ will solve our boundary value problem provided

$$
P\left[T 2^{-p-1} \sigma_{k} \zeta \bar{\delta} \int_{\partial M} \phi_{p} *\left(\omega_{p+1}\right)^{-}\right]+\frac{1}{2} T \zeta \phi_{p}=\theta_{k+p}
$$

on $\partial M$. We seek therefore to determine $\phi_{p}$ as a solution of the singular integral equation (2).

The solution of the integral equation depends upon a discussion of the transposed equation [11]. The transposed, or associated, integral equation arises when we attempt to solve in the exterior of $M$ the boundary value problem

$$
\begin{aligned}
\bar{d} \bar{\delta} B_{p+1} & =0 \\
T * B_{p+1} & =\left(\theta_{2 k-p-1}\right)^{-}
\end{aligned}
$$

on $\partial M$,

in the form

$$
B_{p+1}=2^{-p-1} \sigma_{k} \bar{d} \int_{\partial M}\left(* \phi_{p+1}\right)\left(\omega_{p}\right)^{-} .
$$

Indeed, $T * \bar{\delta} \omega_{p+2}=2^{-1}(-1)^{p+1} T d * \omega_{p+2}$ and

$$
T * 2^{-p-2} \sigma_{k} \bar{\delta} \int_{\partial M} \phi_{p+1} *\left(\omega_{p+2}\right)^{-}
$$

is continuous across $\partial M$, while

$$
T * 2^{-p-1} \sigma_{k} \bar{d} \int_{\partial M}\left(* \phi_{p+1}\right)\left(\omega_{p}\right)^{-}
$$


must jump by $T * \phi_{p+1}$ on $\partial M$. Therefore, the boundary values of $T * B_{p+1}$ on $\partial M$ from the exterior of $M$ are

$$
T * B_{p+1}=P\left[T 2^{-p-1} \sigma_{k} * \bar{d} \int_{\partial M}\left(* \phi_{p+1}\right)\left(\omega_{p}\right)^{-}\right]-\frac{1}{2} T * \phi_{p+1} .
$$

Noting that $(-2)^{k} * \phi_{p+1}=\left[*\left(\zeta \phi_{p+1}\right)\right] \vec{\zeta}$, we obtain for the determination of $\phi_{p+1}$ the singular integral equation

$$
\begin{aligned}
P\left[T 2^{-p-1} \sigma_{k} * \int_{\partial M}\left(* \zeta \phi_{p+1}\right) \bar{\zeta}\left(\bar{\delta} \omega_{p+1}\right)^{-}\right]+\frac{1}{2} T\left[\left(* \zeta \phi_{p+1}\right) \bar{\zeta}\right] \\
=-(-2)^{k}\left(\theta_{2 k-p-1}\right)^{-} .
\end{aligned}
$$

The integral equations (2) and (4) to which we are thus led are each the transpose of the other, since the kernel is in the first case

$$
T(z) T(t) \zeta(z) \bar{\delta}(z) *(t) \omega_{p+1}(z, t)
$$

and in the second case

$$
T(z) T(t) *(z)(\zeta(t))^{-}\left(\bar{\delta}(t) \omega_{p+1}(t, z)\right)^{-} .
$$

It follows [11] that (2) can be solved for $\phi_{p}$ if and only if $\theta_{k+p}=T\left(\zeta A_{p}\right)$ is orthogonal to every solution $\phi_{p+1}$ of (4) in the homogeneous case $\theta_{2 k-p-1}=0$, in the sense

$$
\int_{\partial M}\left(* \zeta \phi_{p+1}\right)\left(\theta_{k+p}\right)^{-}=\int_{\partial M}\left(* \zeta \phi_{p+1}\right) \bar{\zeta}\left(A_{p}\right)^{-}=0
$$

or

$$
\int_{\partial M} A_{p *}\left(\phi_{p+1}\right)^{-}=0 .
$$

This result is a consequence of the relation

$$
\begin{aligned}
\int_{\partial M}( & \left.* \zeta \phi_{p+1}\right)\left(\theta_{k+p}\right)^{-} \\
= & P\left[2^{-p-1} \sigma_{k} \int_{\partial M} \int_{\partial M}\left\{*(z) \zeta(z) \phi_{p+1}(z)\right\}(\zeta(z))^{-}\left(\bar{\delta}(z) \phi_{p}(t) *(t) \omega_{p+1}(z, t)\right)^{-}\right] \\
& +\frac{1}{2} \int_{\partial M}\left\{*(z) \zeta(z) \phi_{p+1}(z)\right\}(\zeta(z))^{-}\left(\phi_{p}(z)\right)^{-} \\
= & P\left[\int_{\partial M}\left\{2^{-p-1} \sigma_{k} * \int_{\partial M}\left(* \zeta \phi_{p+1}\right) \bar{\zeta}\left(\bar{\delta} \omega_{p+1}\right)^{-}+\frac{1}{2}\left[* \zeta \phi_{p+1}\right] \zeta\right\}\left(\phi_{p}\right)^{-}\right] \\
= & 0,
\end{aligned}
$$


where $\phi_{p+1}$ runs through precisely the class of solutions of the homogeneous equation (4) and the inner bracket therefore vanishes identically.

We turn now to the study of the solutions $\phi_{p+1}$ of the homogeneous equation (4) in order to analyze the possible boundary forms $\theta_{k+p}$. It is immediately evident that (3) is a form with boundary values $T\left(* B_{p+1}\right)=0$ from the exterior $\tilde{M}$ of $M$. Therefore

$$
2 \int_{\widetilde{M}} \bar{\delta} B_{p+1} *\left(\bar{\delta} B_{p+1}\right)^{-}=-\int_{\partial M}\left(* B_{p+1}\right)\left(\bar{\delta} B_{p+1}\right)^{-}=0,
$$

where the application of Green's theorem in $\tilde{M}$ is justified by the good behavior of $B_{p+1}$ at infinity. Here the minus sign before the integral on the right is a consequence of the relation $\partial \tilde{M}=-\partial M$. We conclude that

$$
\bar{\delta} B_{p+1}=0,
$$

in $\tilde{M}$.

Therefore the form

$$
Q_{p}=2^{-p-1} \sigma_{k} \int_{\partial M}\left(* \phi_{p+1}\right)\left(\omega_{p}\right)^{-}
$$

satisfies the differential equation $\bar{\delta} \bar{d} Q_{p}=0$ in $\tilde{M}$. A second application of Green's theorem yields

$$
\int_{\widetilde{M}} \bar{d} Q_{p}{ }^{*}\left(\bar{d} Q_{p}\right)^{-}=-\int_{\partial M} Q_{p}\left(\bar{d} Q_{p}\right)^{-}=-\int_{\partial M} Q_{p^{*}}\left(B_{p+1}\right)^{-}=0 .
$$

Thus $B_{p+1}=\bar{d} Q_{p}=0$ in $\tilde{M}$.

Since $T * B_{p+1}$ jumps by $T * \phi_{p+1}$ on $\partial M$, we see that $T * B_{p+1}$ has from the interior of $M$ the boundary values

$$
T * B_{p+1}=T * \phi_{p+1} .
$$

Also

$$
\begin{aligned}
\bar{\delta} B_{p+1} & =2^{-p-1} \sigma_{l} \bar{\delta} \bar{d} \int_{\partial M}\left(* \phi_{p+1}\right)\left(\omega_{p}\right)^{-} \\
& =-2^{-p-1} \sigma_{k} \bar{d} \bar{\delta} \int_{\partial M}\left(* \phi_{p+1}\right)\left(\omega_{p}\right)^{-} \\
& =2^{-p-1} \sigma_{k} \bar{d} \int_{\partial M}\left(* \phi_{p+1}\right)\left(\bar{d} \omega_{p-1}\right)^{-} \\
& =(-1)^{p} 2^{-p-1} \sigma_{k} \bar{d} \int_{\partial M} d\left(* \phi_{p+1}\right)\left(\omega_{p-1}\right)^{-}
\end{aligned}
$$

by Stokes' theorem, and since the tangential derivative

$$
T \zeta \bar{d} \omega_{p-1}=(-1)^{k} T d\left(\zeta \omega_{p-1}\right)
$$


is of order of magnitude $r^{-2 k+2}$, we find that

$$
T \zeta \bar{\delta} B_{p+1}=(-1)^{p+k} 2^{-p-1} \sigma_{k} T d\left\{\zeta \int_{\partial M} d\left(* \phi_{p+1}\right)\left(\omega_{p-1}\right)-\right\}
$$

is continuous across $\partial M$. Hence $T \zeta \bar{\delta} B_{p+1}=0$ there.

A final application of Green's theorem gives

$$
2\left(\bar{\delta} B_{p+1}, \bar{\delta} B_{p+1}\right)=\int_{\partial M}\left(* B_{p+1}\right)\left(\bar{\delta} B_{p+1}\right)^{-}=0,
$$

and we conclude that

$$
\bar{\delta} B_{p+1}=0,
$$

Thus $T * \phi_{p+1}=T * B_{p+1}$ is obtained from a form $B_{p+1}$ in the interior of $M$ which satisfies there the differential equations

$$
\bar{d} B_{p+1}=\bar{\delta} B_{p+1}=0 .
$$

Our conclusion is that the boundary expressions $T \zeta A_{p}$ of pure forms $A_{p}$ in $M$ with

$$
\bar{\delta} \bar{d} A_{p}=0, \quad A_{p}=\bar{\delta} Q_{p+1} \quad \text { for some } Q_{p+1},
$$

generate the entire class of forms $\theta_{k+p}=T\left(\zeta C_{p}\right)$ on $\partial M$ such that

$$
\int_{\partial M} C_{p *}\left(B_{p+1}\right)^{-}=0
$$

for every pure $B_{p+1}$ in $M$ with $\bar{d} B_{p+1}=\bar{\delta} B_{p+1}=0$ there.

In order to show that even this restriction is not necessary, we proceed to apply a method of orthogonal projection.

Let $C_{p}$ be any pure form in $M$ with

$$
\left(\bar{d} C_{p}, \bar{d} C_{p}\right)<\infty .
$$

We denote by $\alpha_{p+1}$ the pure form in $M$ such that

$$
\left(\bar{d} C_{p}-\alpha_{p+1}, \bar{d} C_{p}-\alpha_{p+1}\right)=\text { minimum }
$$

under the restriction $\bar{\delta} \bar{d} \alpha_{p+1}=\bar{\delta} \alpha_{p+1}=0$. Since

$$
\Delta \alpha_{p+1}=\bar{\delta} \bar{d} \alpha_{p+1}+\bar{d} \delta \alpha_{p+1}=0,
$$

the coefficients of the competing forms are harmonic functions, and the extremal form $\alpha_{p+1}$ exists.

We make a permissible variation

$$
\alpha_{p+1}+\epsilon \bar{\delta} \omega_{p+2}
$$

of $\alpha_{p+1}$ with singularity outside $M$, and we deduce that the pure form 


$$
E_{p+1}=-2^{-p-1} \sigma_{k}\left(\bar{d} C_{p}-\alpha_{p+1}, \omega_{p+1}\right)
$$

satisfies in the exterior of $M$ the relation

$$
\bar{d} E_{p+1}=2^{-p-1} \sigma_{k}\left(\bar{d} C_{p}-\alpha_{p+1}, \bar{\delta} \omega_{p+2}\right)=0 .
$$

The coefficients of $E_{p+1}$ are clearly harmonic functions there.

On the other hand, we obtain in $M$

$$
\begin{aligned}
\bar{\delta} \bar{d} \bar{\delta} E_{p+1} & =\bar{\delta} \Delta E_{p+1} \\
& =-2^{-p-1} \sigma_{k} \bar{\delta} \Delta \int_{M}\left(\bar{d} C_{p}-\alpha_{p+1}\right) *\left(\omega_{p+1}\right)^{-} \\
& =\bar{\delta}\left(\bar{d} C_{p}-\alpha_{p+1}\right)=\bar{\delta} \bar{d} C_{p},
\end{aligned}
$$

or, better,

$$
\bar{\delta} \bar{d}\left(C_{p}-\bar{\delta} E_{p+1}\right)=0 .
$$

We set, therefore,

$$
A_{p}=C_{p}-\bar{\delta} E_{p+1}
$$

and study $T \zeta A_{p}$ on $\partial M$.

First, it is not hard to see by arguments from potential theory that $\bar{\delta} E_{p+1}$ is continuous across $\partial M[10]$; so also is the tangential derivative $T \zeta \bar{d} \bar{\delta} E_{p+1}$. But outside $M$

$$
\bar{d} \bar{\delta} E_{p+1}=-\bar{\delta} \bar{d} E_{p+1}=0,
$$

and hence $T d\left(\zeta \bar{\delta} E_{p+1}\right)=0$ on $\partial M$. Thus $T \zeta A_{p}$ and $T \zeta C_{p}$ differ on $\partial M$ by a closed form only.

But more can be proved. If $\beta_{p+1}$ is any form in $M$ such that $\bar{d} \beta_{p+1}=\bar{\delta} \beta_{p+1}$ $=0$, we obtain from

$$
\begin{aligned}
\beta_{p+1} & =-2^{-p-1} \sigma_{k} \Delta \int_{M} \beta_{p+1} *\left(\omega_{p+1}\right)^{-} \\
& =2^{-p-1} \sigma_{k} \bar{d} \int_{M} \beta_{p+1} *\left(\bar{d} \omega_{p}\right)^{-}+2^{-p-2} \sigma_{k} \bar{\delta} \int_{M} \beta_{p+1} *\left(\bar{\delta} \omega_{p+2}\right)^{-} \\
& =2^{-p-1} \sigma_{k} \bar{d} \int_{\partial M}\left(* \beta_{p+1}\right)\left(\omega_{p}\right)^{-}+2^{-p-2} \sigma_{k} \bar{\delta} \int_{\partial M} \beta_{p+1} *\left(\omega_{p+2}\right)^{-}
\end{aligned}
$$

the generalized Cauchy formula

$$
\beta_{p+1}=-2^{-p-1} \sigma_{k} \int_{\partial M}\left(* \beta_{p+1}\right)\left(\bar{\delta} \omega_{p+1}\right)^{-}-2^{-p-2} \sigma_{k} \int_{\partial M} \beta_{p+1} *\left(\bar{d} \omega_{p+1}\right)^{-}
$$

Substituting this in the variational relation $\left(\bar{d} C_{p}-\alpha_{p+1}, \beta_{p+1}\right)=0$, we obtain 


$$
\begin{aligned}
& -2^{-p-1} \sigma_{k} \int_{M} \int_{\partial M}\left(\bar{d} C_{p}-\alpha_{p+1}\right) *\left(\beta_{p+1}\right)^{-} * \bar{\delta} \omega_{p+1} \\
& -2^{-p-2} \sigma_{k} \int_{M} \int_{\partial M}\left(\bar{d} C_{p}-\alpha_{p+1}\right)\left(\beta_{p+1}\right)^{-*} * \bar{d} \omega_{p+1} \\
& =-2^{-p-1} \sigma_{k} \int_{\partial M} *\left(\beta_{p+1}\right)-\bar{\delta} \int_{M}\left(\bar{d} C_{p}-\alpha_{p+1}\right) *\left(\omega_{p+1}\right)^{-} \\
& -2^{-p-2} \sigma_{k} \int_{\partial M}\left(\beta_{p+1}\right)^{-} * \bar{d} \int_{M}\left(\bar{d} C_{p}-\alpha_{p+1}\right) *\left(\omega_{p+1}\right)^{-} \\
& =\int_{\partial M} *\left(\beta_{p+1}\right)-\bar{\delta} E_{p+1}+\frac{1}{2} \int_{\partial M}\left(\beta_{p+1}\right)^{-} * \bar{d} E_{p+1}=0 .
\end{aligned}
$$

But since $\bar{d} E_{p+1}$ vanishes on $\partial M$, this yields

$$
\int_{\partial M} \bar{\delta} E_{p+1} *\left(\beta_{p+1}\right)-=0
$$

for every $\beta_{p+1}$ in $M$ with $\bar{d} \beta_{p+1}=\bar{\delta} \beta_{p+1}=0$.

The method of orthogonal projection therefore gives a form $A_{p}$ such that $T \zeta A_{p}$ differs from the arbitrary values $T \zeta C_{p}$ by a form $T \zeta \bar{\delta} E_{p+1}$ which satisfies the orthogonality relation

$$
\int_{\partial M} \bar{\delta} E_{p+1} *\left(\beta_{p+1}\right)^{-}=0 .
$$

This condition is precisely the one which we developed with the procedure of integral equations, and thus our previous theory shows that $T \zeta \bar{\delta} E_{p+1}$ is a permissable boundary form for our Dirichlet problem. A combination of the methods leads us to conclude that it is always possible to find a solution of the given boundary value problem. The only assumption required is that the given boundary values $T \zeta C_{p}$ be generated by a form expressible as $C_{p}$ $=\bar{\delta} R_{p+1}$, so that the solution $A_{p}$ has also the form $A_{p}=\bar{\delta} Q_{p+1}$.

Uniqueness of the solution is quite elementary. For if $T \zeta A_{p}=0$ on $\partial M$, then

$$
\left(\bar{d} A_{p}, \bar{d} A_{p}\right)=\int_{\partial M} A_{p} *\left(\bar{d} A_{p}\right)^{-}=0,
$$

so that $\bar{d} \bar{\delta} Q_{p+1}=\bar{d} A_{p}=0$. Hence

$$
\left(\bar{\delta} Q_{p+1}, \bar{\delta} Q_{p+1}\right)=\frac{1}{2} \int_{\partial M}\left(* Q_{p+1}\right)\left(\bar{\delta} Q_{p+1}\right)^{-}=\frac{1}{2} \int_{\partial M}\left(* Q_{p+1}\right)\left(A_{p}\right)^{-}=0
$$

and $A_{p}=\bar{\delta} Q_{p+1}=0$ in $M$. 
A quite analogous treatment can be given for the related Neumann boundary value problem

$$
\begin{aligned}
& \overline{d \delta} B_{p+1}=0, \\
& \text { in } M \text {, } \\
& T * B_{p+1}=\left(\theta_{2 k-p-1}\right)^{-} \text {, } \\
& B_{p+1}=\bar{d} Q_{p}
\end{aligned}
$$

and existence and uniqueness of the solution $B_{p+1}$ can be obtained. In this case, of course, the Dirichlet integral is

$$
\left(\bar{\delta} B_{p+1}, \bar{\delta} B_{p+1}\right) \text {. }
$$

4. Green's form, Neumann's form, and the kernel form. The existence theorems of the previous section show that we can define a pure Green's $p$-form $G_{p}(z, t)$ and a pure Neumann's $p$-form $N_{p}(z, t)$ in $M$ as follows. In $M$

$$
\begin{gathered}
G_{p}(z, t)=2^{-p-1} \sigma_{k} \bar{\delta}\left(\omega_{p+1}+\text { regular terms }\right), \\
\bar{\delta} \bar{d} G_{p}(z, t)=0,
\end{gathered}
$$

while on $\partial M$

$$
T \zeta G_{p}(z, t)=0 .
$$

In $M$

$$
\begin{gathered}
N_{p}(z, t)=2^{-p+1} \sigma_{k} \bar{d}\left(\omega_{p-1}+\text { regular terms }\right), \\
\bar{d} \delta N_{p}(z, t)=0
\end{gathered}
$$

and on $\partial M$

$$
T * N_{p}(z, t)=0 .
$$

These conditions determine $G_{p}$ and $N_{p}$ uniquely.

The regular kernel form

$$
K_{p}(z, t)=\bar{d} G_{p-1}(z, t)+\bar{\delta} N_{p+1}(z, t)
$$

clearly satisfies the analyticity relations $\bar{d} K_{p}=\bar{\delta} K_{p}=0$ in $M$, and if $\beta_{p}$ also satisfies $\bar{d} \beta_{p}=\bar{\delta} \beta_{p}=0$, we obtain

$$
\begin{aligned}
\left(\beta_{p}, K_{p}\right) & =\left(\beta_{p}, \bar{d} G_{p-1}\right)+\left(\beta_{p}, \bar{\delta} N_{p+1}\right) \\
& =\beta_{p}+\int_{\partial M}\left(* \beta_{p}\right)\left(G_{p-1}\right)^{-}+\frac{1}{2} \int_{\partial M} \beta_{p} *\left(N_{p+1}\right)^{-}
\end{aligned}
$$

from Green's theorem and the Cauchy formula. Thus $K_{p}$ has the characteristic reproducing property

$$
\beta_{p}=\left(\beta_{p}, K_{p}\right)
$$

and is symmetric, 


$$
K_{p}(z, t)=\left(K_{p}(t, z)\right)^{-}=\kappa_{i_{1}} \cdots i_{p}, j_{1} j_{2} \cdots j_{p} d z_{i_{1}} \cdots d z_{i_{p}} \cdot d \bar{t}_{j_{1}} \cdots d \bar{t}_{j_{p}}
$$
with

If $\beta_{p}^{(\nu)}(z)$ is any complete orthogonal system for the class of pure forms $\beta_{p}$

$$
\bar{d} \beta_{p}=\bar{\delta} \beta_{p}=0, \quad\left(\beta_{p}, \beta_{p}\right)<\infty,
$$

in the sense

$$
\left(\beta_{p}^{(\nu)}, \beta_{p}^{(\mu)}\right)=\delta_{\nu \mu}= \begin{cases}0, & \nu \neq \mu, \\ 1, & \nu=\mu,\end{cases}
$$

then we obtain in the usual manner the Bergman expansion

$$
K_{p}(z, t)=\sum_{p=1}^{\infty} \beta_{p}^{(v)}(z)\left(\beta_{p}^{(v)}(t)\right)^{-}
$$

In particular, the coefficient

$$
\bar{\kappa}_{0}=2{ }^{k} \kappa_{12} \ldots k, 12 \cdots k
$$

is the well known analytic kernel function of the $k$ complex variables $z_{1}, \cdots, z_{k}$ in $M[1]$.

It is of interest to reverse our point of view and obtain the Green's and Neumann's forms $G_{p-1}, N_{p+1}$ from the kernel form $K_{p}$. We note that $K_{p}$ is the form $\beta_{p}$ minimizing

$$
\left(\beta_{p}, \beta_{p}\right)
$$

for fixed $\beta_{p}$ at $z=t$, under the differential restraints $\bar{d} \beta_{p}=\bar{\delta} \beta_{p}=0$. An application of the theory of the problem of Bolza in the calculus of variations tells us, therefore, that there is a Lagrange multiplier $\lambda$ and two pure Lagrange multiplier forms $\lambda_{p-1}$ and $\mu_{p+1}$ in $M$ such that

$$
\left(K_{p}, h_{p}\right)+\lambda\left(h_{p}\right)^{-}+\left(\lambda_{p-1}, \bar{\delta} h_{p}\right)+\left(\mu_{p+1}, \bar{d} h_{p}\right)=0
$$

for every variation $h_{p}$ of $K_{p}$. If we take $h_{p}=\beta_{p}$ with $\bar{d} \beta_{p}=\bar{\delta} \beta_{p}=0$, the reproducing property of the kernel yields $\lambda=-1$. Green's theorem gives

$$
\left(K_{p}-\frac{1}{2} \bar{d} \lambda_{p-1}-2 \bar{\delta} \mu_{p+1}, h_{p}\right)+\int_{\partial M}\left[\frac{1}{2} \lambda_{p-1} *\left(h_{p}\right)^{-}+\left(* \mu_{p+1}\right)\left(h_{p}\right)^{-}\right]=0,
$$

and from the arbitrary nature of $h_{p}$, we obtain

$$
\begin{aligned}
K_{p} & =\frac{1}{2} \bar{d} \lambda_{p-1}+2 \bar{\delta} \mu_{p+1}, & & \text { in } M, \\
T \zeta \lambda_{p-1} & =T * \mu_{p+1}=0, & & \text { on } \partial M .
\end{aligned}
$$

Thus $\lambda_{p-1}=2 G_{p-1}$ and $\mu_{p+1}=(1 / 2) N_{p+1}$, whence we have an interpretation of the Green's and Neumann's forms as Lagrange multipliers for the extremal 
problem of Bolza type which characterizes the kernel $K_{p}$. This formal procedure serves to motivate our earlier discussion of boundary value problems involving $p$-forms.

We turn next to the representation of the solution of the boundary value problems in terms of $G_{p}$ and $N_{p}$. We shall confine ourselves to the Dirichlet problem as solved by the Green's form.

By definition of $G_{p}$, there is a form

$$
\Gamma_{p+1}=2^{-p-1} \sigma_{k} \omega_{p+1}+\text { regular terms }
$$

in $M$ such that $G_{p}=\bar{\delta} \Gamma_{p+1} . \Gamma_{p+1}$ is uniquely determined by the requirement

$$
\left(\Gamma_{p+1}, h_{p+1}\right)=0
$$

for every $h_{p+1}$ such that $\bar{\delta} h_{p+1}=0$. If $\bar{\delta} \bar{d} A_{p}=0$ in $M$, and if $A_{p}=\bar{\delta} Q_{p+1}$ for a uniquely determined form $Q_{p+1}$ with

$$
\left(Q_{p+1}, h_{p+1}\right)=0
$$

whenever $\bar{\delta} h_{p+1}=0$, then we obtain

$$
\begin{aligned}
\int_{\partial M} A_{p} * & \left(\Gamma_{p+1}\right)^{-}-2^{-p-2} \sigma_{k} \int_{\partial M} Q_{p+1}\left(\bar{d} \omega_{p+1}\right)^{-} \\
= & \int_{\partial M} A_{p *}\left(\Gamma_{p+1}\right)^{-}-\int_{\partial M}\left(* Q_{p+1}\right)\left(\bar{\delta} \Gamma_{p+1}\right)^{-}-2^{-p-2} \sigma_{k} \int_{\partial M} Q_{p+1}\left(\bar{d} \omega_{p+1}\right)^{-} \\
= & \left(\bar{d} A_{p}, \Gamma_{p+1}\right)+2\left(\bar{\delta} Q_{p+1}, \bar{\delta} \Gamma_{p+1}\right)-2\left(\bar{\delta} Q_{p+1}, \bar{\delta} \Gamma_{p+1}\right) \\
& -\left(Q_{p+1}, \bar{d} \delta \Gamma_{p+1}\right)-2^{-p-1} \sigma_{k}\left(Q_{p+1}, \bar{\delta} \bar{d} \omega_{p+1}\right) \\
& -2^{-p-2} \sigma_{k}\left(\bar{d} Q_{p+1}, \bar{d} \omega_{p+1}\right)+Q_{p+1} \\
= & -\left(Q_{p+1}, \bar{d} \delta\left[\Gamma_{p+1}-2^{-p-1} \sigma_{k} \omega_{p+1}\right]\right) \\
& +2^{-p-2} \sigma_{k} \bar{\delta}\left(\bar{d} Q_{p+1}, \omega_{p+2}\right)+Q_{p+1} \\
= & 2^{-p-2} \sigma_{k} \bar{\delta}\left(\bar{d} Q_{p+1}, \omega_{p+2}\right)+Q_{p+1} .
\end{aligned}
$$

By application of the operator $\bar{\delta}$ to this identity, we derive the formula

$$
A_{p}=\bar{\delta} \int_{\partial M} A_{p} *\left(\Gamma_{p+1}\right)^{-}
$$

for the solution of the Dirichlet problem.

The corresponding formula for the solution of the Neumann problem is

$$
B_{p+1}=\frac{1}{2} \bar{d} \int_{\partial M}\left(* B_{p+1}\right)\left(H_{p}\right)^{-},
$$

where

$$
H_{p}=2^{-p} \sigma_{k} \omega_{p}+\text { regular terms }
$$


is the unique form such that $N_{p+1}=\bar{d} H_{p}$ and such that

$$
\left(H_{p}, h_{p}\right)=0
$$

whenever $\bar{d} h_{p}=0$.

The symmetry of the Green's form $G_{p}(z, t)$ is obtained in a similar way. We have

$$
\begin{aligned}
\int_{\partial M} \bar{\delta} \Gamma_{p+1} *\left(\Gamma_{p+1}\right)^{-} & -\int_{\partial M}\left(* \Gamma_{p+1}\right)\left(\bar{\delta} \Gamma_{p+1}\right)^{-} \\
& -2^{-p-2} \sigma_{k} \int_{\partial M} \Gamma_{p+1} *\left(\bar{d} \omega_{p+1}\right)^{-}+2^{-p-2} \sigma_{k} \int_{\partial . M}\left(* \bar{d} \omega_{p+1}\right)\left(\Gamma_{p+1}\right)^{-} \\
= & \Gamma_{p+1}-\left(\Gamma_{p+1}\right)^{-}+2^{-p-2} \sigma_{k}\left(\bar{d} \omega_{p+1}, \bar{d} \Gamma_{p+1}\right) \\
& -2^{-p-2} \sigma_{k}\left(\bar{d} \Gamma_{p+1}, \bar{d} \omega_{p+1}\right)
\end{aligned}
$$

so that application of the operator $\bar{\delta}(z)(\bar{\delta}(t))^{-}$yields the rule

$$
(\bar{\delta}(t))^{-} G_{p}(z, t)=\bar{\delta}(z)\left(G_{p}(t, z)\right)^{-} .
$$

Likewise, by the analogous argument for Neumann's problem,

$$
(\bar{d}(t))-N_{p}(z, t)=\bar{d}(z)\left(N_{p}(t, z)\right)^{-} .
$$

The boundary value problems can be reformulated as orthogonal decomposition theorems in the Hilbert space $L^{2}$ of pure forms $C_{p}=\bar{\delta} R_{p+1}$ with

$$
\left(\bar{d} C_{p}, \bar{d} C_{p}\right)<\infty
$$

and the Hilbert space $\Lambda^{2}$ of pure forms $D_{p}=\bar{d} S_{p-1}$ with

$$
\left(\bar{\delta} D_{p}, \bar{\delta} D_{p}\right)<\infty,
$$

and a Dirichlet principle can be stated. We introduce for this purpose the subclass of $L^{2}$ generated by elements $\Phi_{p}$ such that

$$
T \zeta \Theta_{p}=0,
$$

on $\partial M$,

and the subclass of $\Lambda^{2}$ generated by elements $\Psi_{p}$ such that

$$
T * \Psi_{p}=0
$$

Then for each $A_{p} \in L^{2}$ with $\bar{\delta} \bar{d} A_{p}=0$ in $M$, we have the orthogonality relation

$$
\left(\bar{d} \Phi_{p}, \bar{d} A_{p}\right)=\int_{\partial M} \Phi_{p} *\left(\bar{d} A_{p}\right)^{-}=0,
$$

whereas for each $B_{p} \in \Lambda^{2}$ with $\bar{d} \bar{\delta} B_{p}=0$ in $M$, we obtain

$$
\left(\bar{\delta} \Psi_{p}, \bar{\delta} B_{p}\right)=\frac{1}{2} \int_{\partial M}\left(* \Psi_{p}\right)\left(\bar{\delta} B_{p}\right)^{-}=0 .
$$


Thus it follows from our theory of the Dirichlet problem for $\bar{\delta} \bar{d} A_{p}=0$ and the Neumann problem for $\bar{d} \bar{\delta} B_{p}=0$ that each $C_{p} \in L^{2}$ has a unique orthogonal decomposition

$$
C_{p}=A_{p}+\Phi_{p}
$$

and each $D_{p} \in \Lambda^{2}$ has a unique orthogonal decomposition

$$
D_{p}=B_{p}+\Psi_{p}
$$

The Dirichlet principle is an immediate consequence of these decompositions. It states that for given $C_{p}$, the form $A_{p}$ such that $T \zeta A_{p}=T \zeta C_{p}$ on $\partial M$ and

$$
\left(\bar{d} A_{p}, \bar{d} A_{p}\right)=\text { minimum }
$$

satisfies the partial differential equations $\bar{\delta} \bar{d} A_{p}=0$. Similarly, for given $D_{p}$, the form $B_{p}$ such that $T * B_{p}=T * D_{p}$ on $\partial M$ and

$$
\left(\bar{\delta} B_{p}, \bar{\delta} B_{p}\right)=\text { minimum }
$$

satisfies the system of equations $\bar{d} \bar{\delta} B_{p}=0$.

We close this section with a remark concerning the generalized Cauchy formula

$$
\beta_{p}=-2^{-p} \sigma_{k} \int_{\partial M}\left(* \beta_{p}\right)\left(\bar{\delta} \omega_{p}\right)^{-}-2^{-p-1} \sigma_{k} \int_{\partial M} \beta_{p^{*}}\left(\bar{d} \omega_{p}\right)^{-},
$$

valid for pure forms $\beta_{p}$ such that $\bar{d} \beta_{p}=\bar{\delta} \beta_{p}=0$ in $M$. If one introduces the class of pure forms $U_{p}$ which satisfy in $M$ the Laplace equation $\Delta U_{p}=0$, one obtains the identity

$$
\begin{aligned}
\int_{\partial M}\left(* \beta_{p}\right)\left(\bar{\delta} U_{p}\right)^{-}+\frac{1}{2} \int_{\partial M} \beta_{p} * & \left(\bar{d} U_{p}\right)^{-} \\
& =2\left(\bar{\delta} \beta_{p}, \bar{\delta} U_{p}\right)+\frac{1}{2}\left(\bar{d} \beta_{p}, \bar{d} U_{p}\right)+\left(\beta_{p}, \Delta U_{p}\right)=0,
\end{aligned}
$$

for each $U_{p}$, when $\bar{d} \beta_{p}=\bar{\delta} \beta_{p}=0$. Conversely, suppose $\beta_{p}$ is defined on $\partial M$ and satisfies

$$
\int_{\partial M}\left(* \beta_{p}\right)\left(\bar{\delta} U_{p}\right)^{-}+\frac{1}{2} \int_{\partial M} \beta_{p} *\left(\bar{d} U_{p}\right)^{-}=0
$$

for each $U_{p}$ with $\Delta U_{p}=0$ in $M$. If we extend $\beta_{p}$ into $M$ as a solution of Laplace's equation $\Delta \beta_{p}=0$, we obtain

$$
2\left(\bar{\delta} \beta_{p}, \bar{\delta} U_{p}\right)+\frac{1}{2}\left(\bar{d} \beta_{p}, \bar{d} U_{p}\right)=0 .
$$


For $U_{p}=\beta_{p}$, this yields $\bar{d} \beta_{p}=\bar{\delta} \beta_{p}=0$. Thus a necessary and sufficient condition that a pure form $\beta_{p}$ defined on $\partial M$ represent the boundary values of a form in $M$ satisfying $\bar{\delta} \beta_{p}=\bar{d} \beta_{p}=0$ is that

$$
\int_{\partial M}\left(* \beta_{p}\right)\left(\bar{\delta} U_{p}\right)^{-}+\frac{1}{2} \int_{\partial M} \beta_{p} *\left(\bar{d} U_{p}\right)^{-}=0
$$

for each solution $U_{p}$ of Laplace's equation in $M$. If the equations $\bar{d} \beta_{p}=\bar{\delta} \beta_{p}$ $=0$ are viewed as a generalization of the Cauchy-Riemann equations, this result can be interpreted as a generalized Cauchy theorem.

A similar reasoning shows that if $\Delta U_{p}=0$ in $M$ and $T \zeta \bar{\delta} U_{p}=T * \bar{d} U_{p}=0$ on $\partial M$, then $\bar{d} U_{p}=\bar{\delta} U_{p}=0$ in $M$.

5. Special cases and examples. It is evident that the Dirichlet problem for forms $A_{p}$ with $\bar{\delta} \bar{d} A_{p}=0$ is equivalent to the Neumann problem for forms $B_{k-p}$ with $\bar{d} \bar{\delta} B_{k-p}=0$. We indicate here the significance of these problems for some special values of $p$ and $k$.

For $p=0$, the Dirichlet problem is that for the classical Laplace equation in $2 k$ real variables, and our work adds nothing to the known theory. But for $p=k-1$, we obtain the most important case of our theory and develop through integral equations the recently discovered formalism of boundary value problems for the Cauchy-Riemann equations in $k$ complex variables [8]. Indeed, here with forms $\beta_{k}=\beta_{p+1}$ the equation $\bar{d} \beta_{p}=0$ is vacuous, while the relation $\bar{\delta} \beta_{k}=0$ represents precisely the Cauchy-Riemann equations for the single coefficient $b_{i_{1}} \ldots i_{k}$ of

$$
\beta_{k}=b_{i_{1}} \cdots i_{k} d z_{i_{1}} \cdots d z_{i_{k}},
$$

which must therefore be analytic in the $k$ complex variables $z_{1}, \cdots, z_{k}$. The Neumann's form $N_{k+1}$ is absent, and the Green's form $G_{p}=G_{k-1}$ can be taken as the natural generalization of the Green's function in one variable, by virtue of the kernel identity

$$
K_{k}=\bar{d} G_{k-1} .
$$

An even more direct generalization appears in terms of the form $\Gamma_{k}$ with $\bar{\delta} \Gamma_{k}=G_{k-1}$, and the kernel identity can also be written

$$
K_{k}=\bar{d} \bar{\delta} \Gamma_{k}=\Delta \Gamma_{k} .
$$

For further discussion the reader is referred to previous papers [9]. However, the formulation of the theory given here has special interest because it introduces the method of the Fredholm integral equation, thus completing the application of the apparatus of elliptic partial differential equations to the study of functions of several complex variables.

Since we are using the notation of the exterior differential calculus of $E$. Cartan, it is worthwhile to compare at this point the complex operators $\bar{d}, \bar{\delta}$ and Hodge's operators $d, \delta$. The distinct advantage of our operators is that 
they yield not only Laplace's equation when $p=0$, but also the CauchyRiemann equations for $k$ complex variables when $p=k$. Note, however, that Hodge's harmonic forms have applications in magnetostatics for three dimensions, etc. It is, of course, true that all our results have a direct analogue for Hodge's forms in $k$-dimensional space. In particular, the kernel form can be introduced in that theory and the method of kernel orthogonal projection goes through, but it seems unnecessary to mention details here, since the case of the complex operators $\bar{d}, \bar{\delta}$ serves to present the leading ideas.

It is of particular interest to write down the fundamental integral equation (2) when $p=0, k=1$, the classical case. The boundary $\partial M$ is a curve in the complex $z$-plane with unit tangent $z^{\prime}(s)$, and we obtain

$$
\frac{1}{2 \pi i} \oint_{\partial M} \frac{\phi(z) d z}{z-t}+\frac{1}{2} \phi(t)=\theta(t)
$$

for the interior Dirichlet problem. Such singular integral equations of Hilbert type have been amply treated in the literature [14]. The homogeneous transposed equation is

$$
\frac{\left(z^{\prime}(s)\right)^{-}}{2 \pi i} \oint_{\partial M} \frac{\psi(t) d s}{\bar{t}-\bar{z}}+\frac{1}{2} \psi(z)=0,
$$

which has eigenfunctions of the form $\psi(z)=\left(g(z) z^{\prime}(s)\right)^{-}$, with $g(z)$ analytic in $M$. Thus one obtains from a discussion of this integral equation the orthogonal decomposition

$$
\theta(z)=f(z)+\left(g(z) z^{\prime}(s)\right)^{-}
$$

of any square integrable function $\theta(z)$, defined on the curve $\partial M$, in terms of functions $f(z)$ and $g(z)$ analytic in $M$. This decomposition is basic in a discussion of the Szegö kernel function and was treated originally by an alternative method of conformal mapping $[10 ; 16]$.

An intermediate case of the generalized Cauchy-Riemann equations $\bar{d} \beta_{p+1}=\bar{\delta} \beta_{p+1}=0$ which has special importance occurs when $p=0, k=2$. Here we have

$$
\beta_{1}=a_{1} d z_{1}+a_{2} d z_{2}
$$

and the equations $\bar{d} \beta_{1}=\bar{\delta} \beta_{1}=0$ can be written

$$
\frac{\partial a_{1}}{\partial \bar{z}_{1}}=-\frac{\partial a_{2}}{\partial \bar{z}_{2}}, \quad \frac{\partial a_{1}}{\partial z_{2}}=\frac{\partial a_{2}}{\partial z_{1}} .
$$

These will be recognized as the equations of Fueter $[6 ; 7]$ for quaternion functions. Thus we obtain as a particular case his theory, which was the first to center about a Cauchy formula of the type given in the present work.

Returning to the original boundary value problem 


$$
\begin{aligned}
T \zeta A_{p} & =\theta_{k+p}, \\
\bar{\delta} \bar{d} A_{p} & =0, \\
A_{p} & =\bar{\delta} Q_{p+1},
\end{aligned}
$$

given on $\partial M$, in $M$, in $M$,

we note that it is precisely the last, less usual, condition which makes the solution unique, with coefficients which are analytic as functions of real variables. Thus we might say that this condition gives a strongly elliptic system of partial differential equations, whereas without this condition the problem is only weakly elliptic. One has, in fact,

$$
\Delta A_{p}=\bar{\delta} \bar{d} A_{p}+\bar{d} \bar{\delta} \bar{\delta} Q_{p+1}=0,
$$

so that the coefficients of $A_{p}$ are harmonic functions, and our boundary value problem can be formulated as a question of the determination of a system of harmonic functions satisfying certain differential relations and correspondingly fewer boundary conditions than usual.

We shall discuss here briefly the weakly elliptic system

$$
\bar{\delta} \bar{d} A_{p}=0
$$

for the case $p=1, k=2$ in a product region $M$, and we shall prove that a solution can be found for prescribed $A_{p}$ on $\partial M$, and not just for prescribed $T \zeta A_{p}$. The solution is not, however, unique or regular, since any form $A_{p}$ $=\bar{d} Q_{p-1}$ satisfies the equation. Our result is not restricted, of course, to $k=2$, but holds in general for $p=k-1$, as can be shown by a somewhat more involved reasoning.

To solve $\bar{\delta} \bar{d} A_{1}=0$ for $k=2$, we recall that the method of orthogonal projection yields for given pure $C_{1}$ in the product region $M$ a form

$$
E_{2}=-2^{-2} \sigma_{2}\left(\bar{d} C_{1}-\alpha_{2}, \omega_{2}\right),
$$

with $\bar{d} E_{2}=0$ outside $M$, and with $\bar{\delta} \bar{d} \bar{\delta} E_{2}=\bar{\delta} \bar{d} C_{1}$ in $M$. Since $k=2$, the relation $\bar{d} E_{2}=0$ outside $M$ is trivial, and also $\bar{d} \bar{\delta} E_{2}=0$ is immediate. If we can construct outside $M$ a form $F_{0}$ such that $\bar{d} F_{0}=\bar{\delta} E_{2}$, then we can find the desired form $A_{1}$. For we can extend $F_{0}$ smoothly into $M$, and we can set

$$
A_{1}=C_{1}-\bar{\delta} E_{2}+\bar{d} F_{0} \text {. }
$$

Then

$$
\bar{\delta} \bar{d} A_{1}=\delta \bar{d} C_{1}-\bar{\delta} \bar{d} \bar{\delta} E_{2}+\bar{\delta} \bar{d} \bar{d} F_{0}=0
$$

in $M$, and $A_{1}=C_{1}$ on $\partial M$ by the continuity of $\bar{\delta} E_{2}$ and $\bar{d} F_{0}$ across $\partial M$, since $\bar{\delta} E_{2}=\bar{d} F_{0}$ outside $M$.

We turn, then, to the construction of $F_{0}$, a question related to the problem of Cousin for analytic functions of two complex variables. We note that

$$
\bar{\delta} E_{2}=2^{-2} \sigma_{2}\left(\bar{d} C_{1}-\alpha_{2}, \bar{d} \omega_{1}\right),
$$


where

$$
\bar{d} \omega_{1}=-\frac{\partial r^{-2}}{\partial t_{2}} d t_{1} d t_{2} d \bar{z}_{1}+\frac{\partial r^{-2}}{\partial t_{1}} d t_{1} d t_{2} d \bar{z}_{2}
$$

But

$$
\begin{aligned}
& \frac{\partial r^{-2}}{\partial t_{2}}=-\frac{\partial r^{-2}}{\partial z_{2}}=\frac{\partial}{\partial \bar{z}_{1}}\left\{\lambda r^{-2} \frac{\bar{z}_{1}-\bar{t}_{1}}{z_{2}-t_{2}}+(\lambda-1) r^{-2} \frac{\bar{z}_{2}-\bar{t}_{2}}{z_{1}-t_{1}}\right\}, \\
& \frac{\partial r^{-2}}{\partial t_{1}}=-\frac{\partial r^{-2}}{\partial z_{1}}=-\frac{\partial}{\partial \bar{z}_{2}}\left\{\lambda r^{-2} \frac{\bar{z}_{1}-\bar{t}_{1}}{z_{2}-t_{2}}+(\lambda-1) r^{-2} \frac{\bar{z}_{2}-\bar{t}_{2}}{z_{1}-t_{1}}\right\}
\end{aligned}
$$

for any $\lambda$, and hence

$$
\bar{d}(t) \omega_{1}(t, z)=-(\bar{d}(z))^{-}\left\{\lambda r^{-2} \frac{\bar{z}_{1}-\bar{t}_{1}}{z_{2}-t_{2}}+(\lambda-1) r^{-2} \frac{\bar{z}_{2}-\bar{t}_{2}}{z_{1}-t_{1}}\right\} d t_{1} d t_{2} .
$$

'Thus,

$$
\bar{\delta} E_{2}=-\bar{d} 2^{-2} \sigma_{2}\left(\bar{d} C_{1}-\alpha_{2},\left\{\lambda r^{-2} \frac{\bar{z}_{1}-\bar{t}_{1}}{z_{2}-t_{2}}+(\lambda-1) r^{-2} \frac{\bar{z}_{2}-\bar{t}_{2}}{z_{1}-t_{1}}\right\} d t_{1} d t_{2}\right) .
$$

The coefficient of $\lambda$ depends only on the scalar product

$$
\left(\bar{d} C_{1}-\alpha_{2}, \frac{d t_{1} d t_{2}}{\left(z_{1}-t_{1}\right)\left(z_{2}-t_{2}\right)}\right),
$$

which can be shown to vanish by a variational argument when $z_{1}$ is not in the projection $M_{1}$ of $M$ on the $z_{1}$-plane and $z_{2}$ is not in the projection $M_{2}$ of $M$ on the $z_{2}$-plane, by virtue of the extremal property of $\alpha_{2}$. Therefore, we can take

$$
F_{0}=-2^{-2} \sigma_{2}\left(\bar{d} C_{1}-\alpha_{2},\left\{\lambda r^{-2} \frac{\bar{z}_{1}-\bar{t}_{1}}{z_{2}-t_{2}}+(\lambda-1) r^{-2} \frac{\bar{z}_{2}-\bar{t}_{2}}{z_{1}-t_{1}}\right\} d t_{1} d t_{2}\right),
$$

with $\lambda=1$ when $z_{1}$ is in $M_{1}$ and $\lambda=0$ when $z_{2}$ is in $M_{2}$, and with arbitrary $\lambda$ in all other cases. This gives, indeed, a uniquely defined function $F_{0}$ outside $M$, since $F_{0}$ is independent of $\lambda$ when $z_{1}$ is outside $M_{1}$ and $z_{2}$ is outside $M_{2}$. Thus our discussion of the boundary value problem is completed.

An analogous argument could be given when $M$ is no longer a product region if it were true that $\bar{d} \phi_{p}=0$ implies $\phi_{p}=\bar{d} \psi_{p-1}$ in the large. However, the example $\phi_{p}=\bar{\delta} \omega_{k}$ shows that this is not the case.

\section{REFERENCES}

1. S. Bergman, Sur les fonctions orthogonales de plusieurs variables complexes, Mémorial des Sciences Mathématiques, vol. 106, Paris, 1947. 
2. - The kernel function and conformal mapping, Mathematical Surveys, no. 5, New York, American Mathematical Society, 1950.

3. S. Bergman and M. Schiffer, A representation of Green's and Neumann's functions in the theory of partial differential equations of second order, Duke Math. J. vol. 14 (1947) pp. 609-638.

4. P. Bidal and G. de Rham, Les formes differentielles harmoniques, Comment. Math. Helv. vol. 19 (1946) pp. 1-49.

5. S. Bochner, Analytic and meromorphic continuation by means of Green's formula, Ann. of Math. vol. 44 (1943) pp. 652-673.

6. R. Fueter, Über einen Hartogs'schen Satz, Comment. Math. Helv. vol. 12 (1939) pp. 7580.

7. - Über einen Hartogs'schen Satz in der Theorie der analytischen Funktionen von $n$ komplexen Variablen, Comment. Math. Helv. vol. 14 (1942) pp. 394-400.

8. P. R. Garabedian, $A$ new formalism for functions of several complex variables, Journal d'Analyse Mathématique vol. 1 (1951) pp. 59-80.

9. - A Green's function in the theory of functions of several complex variables, Ann. of Math. vol. 55 (1952) pp. 19-33.

10. P. R. Garabedian and M. Schiffer, On existence theorems of potential theory and conformal mapping, Ann. of Math. vol. 52 (1950) pp. 164-187.

11. G. Giraud, Equations et systèmes d'équations où figurent des valeurs principales d'intégrales, C. R. Acad. Sci. Paris vol. 204 (1937) pp. 628-630.

12. W. V. D. Hodge, A Dirichlet problem for harmonic functionals, with applications to analytic varieties, Proc. London Math. Soc. vol. 36 (1934) pp. 257-303.

13. - The theory and applications of harmonic integrals, Cambridge, 1941.

14. F. Noether, Über eine Klasse singulärer Integralgleichungen, Math. Ann. vol. 82 (1921) pp. 42-63.

15. M. Schiffer, The kernel function of an orthonormal system, Duke Math. J. vol. 13 (1946) pp. 529-540.

16. - Various types of orthogonalization, Duke Math. J. vol. 17 (1950) pp. 329-366.

17. W. Wirtinger, Zur formalen Theorie der Funktionen von mehr komplexen Veränderlichen, Math. Ann. vol. 97 (1927) pp. 357-375.

Stanford UNiversity,

Stanford, Calif.

Princeton University,

Princeton, N. J. 